\title{
Study on Spontaneous Cerebrospinal Fluid (CSF) Rhinorrhoea: A Birmingham Experience
}

\author{
Sayed MSU' ${ }^{1}$, Dunn $\mathrm{CJ}^{2}$, Alaani $\mathrm{A}^{3}$, Johnson $\mathrm{A}^{4}$
}

\begin{abstract}
Spontaneous Cerebrospinal fluid (CSF) rhinorrhoea is not very common. The aim of this study is to identify the common features in the study group of patients with spontaneous CSF rhinorrhoea, to develop a hypothesis to explain the cause of this condition and to investigate the outcome of surgical techniques adopted to repair the leak. In this retrospective study we have reviewed all the cases of spontaneous CSF leaks attending and receiving treatment from the Department of ENT at Queen Elizabeth Hospital, Birmingham, from 1992 to 2002 .
\end{abstract}

Among the total number of 34 patients with CSF leaks, 15 were spontaneous in nature and formed our study group, of these 15 patients 14 were female; with age range from 37 to 70 years and a median age of 50 years. All the female patients were obese with a body mass index higher than normal range. We tried to identify common factors in the study group and it was evident that female sex, obesity and age play a key role in this condition.

Follow up period ranged from 2 to 98 months and thirteen patients were asymptomatic but two patients remained symptomatic, one of these despite repeated surgical intervention.

Key words: Cerebrospinal Fluid Rhinorrhoea, Endoscopy.

\section{Introduction}

Cerebrospinal fluid rhinorrhoea is a rare cause of watery nasal discharge. It indicates a communication between the nose and the subarachnoid space with breach of bone, dura and arachnoid layers.

It is most commonly traumatic and can occur as a result of disruption of the arachnoid and dura, raised CSF pressure or a bony defect.

Spontaneous CSF rhinorrhoea occurs in 3-4\% of all cases ${ }^{1}$, where normal pressure CSF leaks $(55 \%)$ outnumber the high pressure CSF leaks (45\%).

\footnotetext{
1. Corresponding Author: Mohammad Saleh Uddin Sayed Associate Professor \& Head of ENT Department Nightingale Medical College, Bangladesh

2. Mr. Chris J Dunn

SPR, Queen Elizabeth Hospital

University Hospital Birmingham, England

3. Mr. A Alaani

SPR, Queen Elizabeth Hospital

University Hospital Birmingham, England

4. Mr. Alan Johnson

Consultant, Otolaryngologist

Queen Elizabeth Hospital

University Hospital Birmingham, England
}

Spontaneous CSF rhinorrhoea was first described by Miller $^{2}$, who in 1826 reported a case of boy who had a progressively enlarging head and recurrent bouts of profuse fluid discharge from his nose and the presence of fistula was confirmed at necropsy. As recently as 1969 , fewer than 150 cases had been reported in the literature (Brisman, Huges, and Mount $^{3}$ ). The recent technique of extracranial repair is first described by Dholman ${ }^{4}$, since then many others have described the technique using both flaps and free grafts Wigand $^{5}, 1981$ and Stankiewicz, 1989, were the first to report on endoscopic repair ${ }^{6}$.

High-pressure CSF rhinorrhoea is associated with slow growing tumour $(84 \%)$ or with hydrocephalus where the leak acts as a safety valve to alleviate the increased intracranial pressure. In such cases repair of the leak must be in conjunction with the removal of the underlying pathology ${ }^{7}$.

In normal pressure leaks the following theories of aetiology are described:

a. Ommaya's theory of focal atrophy ${ }^{8}$ states that the contents of the cribiform plate or sella turcica are diminished in bulk due to ischaemia. The empty space becomes a pouch filled with CSF thus enabling the normal CSF pressure pulse to exert a focal and erosive effect.

b. Rupture of the arachnoid sleeves passing through the cribiform plate with olfactory nerve filaments 9

c. Persistent embryonic olfactory lumen ${ }^{9}$.

d. Meningo-encephalocele ${ }^{9}$.

Maldevelopment of diaphragma sellae may be an added factor. Normal CSF pressure is pulsatile and the pulsations are mainly due to respiratory and cardiac activity causing variation of $15-45 \mathrm{~mm}$ of CSF pressure ${ }^{10}$.

Constant pulsations of CSF on the lamina cribriosa cause distension of subarachnoid pouches resulting in erosion of the bone eventually leading to establishment of a fistula ${ }^{11}$. Due to the intimate anatomical relationship between the subarachnoid space and the nasal cavity at lamina cribriosa, perforation of pia arachnoid results in CSF rhinorrhoea ${ }^{11}$.

Historically CSF leaks have been repaired by an intracranial approach. This had the advantage of directly visualising the fistula from above and assessing any co-existing intracranial pathology. Its disadvantages are those of craniotomy with retraction on the frontal lobes causing a high incidence of anosmia, possible cerebral oedema and intracranial haemorrhage with resulting morbidity and mortality ${ }^{12,13}$.

The upper part of the nose especially the area above the superior turbinate is difficult to approach, but with the 
advent of nasendoscope and microscope the area has become accessible and CSF leaks can be identified and repaired successfully with minimum mortality and morbidity. The aim of this study is to identify common features in the group of patients with spontaneous CSF leaks and to develop a hypothesis to explain the cause of this condition and to investigate the outcome of surgical techniques adopted to repair the leak.

\section{Materials and Methods}

In our study we included the patients with spontaneous cerebrospinal fluid rhinorrhoea who attended the outpatient department of Otolaryngology and Head-Neck Surgery at Queen Elizabeth Hospital, Birmingham between September 1992 and November 2002. A total of 34 cases were identified who underwent repair for their CSF leak. Those patients with CSF leak with a history of head injury (traumatic) or with the history of any operation in the base of the skull and nasal roof (iatrogenic), prior to commencement of their CSF rhinorrhoea, were excluded. Fifteen patients were identified as having primary spontaneous CSF rhinorrhoea and were included in this study. Data collection includes patients sex, age, weight, height, BMI, complications, method of localisation of defect, operative techniques, graft materials used during operation, post operative and long term follow up results from the hospital records and telephone interview with the patients.

\section{Results}

Among fifteen cases of primary spontaneous CSF rhinorrhoea thirteen were female and two were male. Their age were ranged from 37 to 70 years with a median age of 50 years. All 13 female patients were overweight (BMI $>24.9$ ) and of these 9 were obese (BMI > 30). One of the two male patients was obese, the remaining male patient was of normal Body Mass Index (BMI - normal = 18.5 $24.9 \mathrm{Kg} / \mathrm{m} 2$ ) shown in figure 1 below.

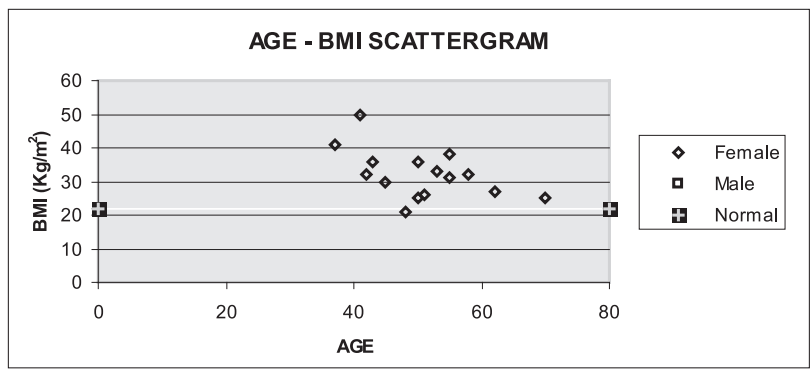

Figure 1: Patient Demographics-Age \& Body Mass Index (BMI)

The Department of Health publishes the U.K BMI distribution for the general population for age and sex groups(http://www.dh.gov.uk/assetRoot/04/06/60/70/04066 070.xls). In the general population, the incidence of obesity (BMI>30), within a similar age matched group to our series, is $27 \%$, however in our group the incidence of obesity was $10 / 15$ or $67 \%$.
Additionally the incidence of obesity in the general population for all females is $22 \%$, our series yielded obesity rates of $9 / 13$ or $70 \%$.

The duration of leak varied from 4 weeks to 6 years. The weight of the patients varied from 70 to 116 KGs and BMI was from 25-50. Four patients had a history of meningitis. One patient presented with left sided hemiparesis with a large pneumoencephalocele in the right fronto parietal region (on the $\mathrm{CT}$ and $\mathrm{MRI}$ ). A frontal burr hole was used to evacuate the air but this reaccumulated.

Two patients were treated for perennial rhinitis for a duration of 4 years before CSF rhinorrhoea was considered. Eight patients had a primary repair and two patients had unsuccessful repair prior to attending our department.

Four patients were identified with a meningocele/ menigoencephalocele in the roof of the nasal cavity and one patient had a nasal glioma. One patient had a large occipital meningioma with signs of increased intracranial pressure and the localising signs of tumour, which was successfully removed by the neurosurgeons by parietal craniotomy with subsequent improvement from the symptoms but she developed spontaneous CSF rhinorrhoea 6 months after she had her initial surgery.

B2 transferrin (Tau protein) was measured in the nasal fluid of eight patients and all were positive. Glucose levels were measured in the nasal fluid in 6 patients and the level was from $3.33 \mathrm{mmol} / \mathrm{L}-3.8 \mathrm{mmol} / \mathrm{L}$. Rigid endoscopy in the outpatient department revealed the leak and pathology in 7 patients.

CT or MRI delineated the bony dehiscence in 10 patients and was reported as normal in 4 patients. Isotope cysternogram was done in one patient where it demonstrated a meningocoele. Intrathecal metrizamide dye was used in one patient which only confirmed the findings of non-contrast CT. CT scan identified ventricular enlargement in one patient who also had a congenital cyst in the septum pellucidum removed by endoscopic cranial surgery through a frontal burr hole. The site of the skull base defect detected per operation is shown in Figure 2 below.

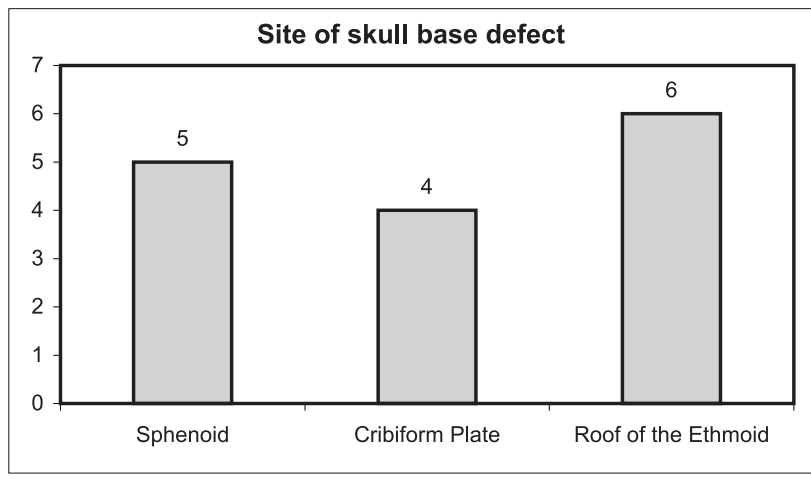

Figure 2: Site of skull base defect

10 patients had undergone endoscopic surgery for their repair of CSF leak, 4 were approached by external ethmoidectomy through Howarth incision. Of the endoscopic group three developed recurrence, and two of these were subsequently approached via an external 
ethmoidectomy. The type of material used for surgical repair of the defect is shown in figure 3 below:

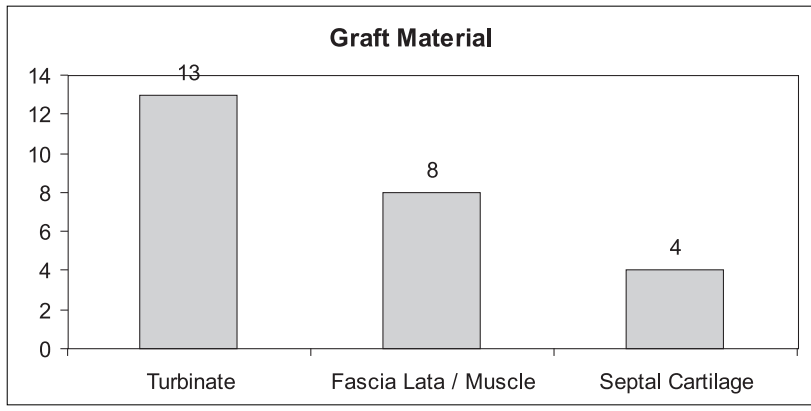

Figure 3: Graft material used for repair

Lyostypt was used in all patients and tisseal in eleven. The nose was packed with Bismuth \& Iodoform Paraffin Paste after the operation and kept in situ for 3-7 days duration depending on the size of repair. Postoperative antibiotics were used in all patients. None had lumbo-peritoneal shunt during operative procedure.

Follow up period was from 2 to 98 months and 13 patients were cured of their symptoms and complications but two are still symptomatic, one of these despite a third attempt at repair.

\section{Discussion}

Obese female patients in their fifth and sixth decades are the most common group to suffer from spontaneous CSF rhinorrhoea. Despite the small sample size with a sex matched sample an F-Test statistical analysis of BMI revealed an $\mathrm{F}$ critical value of 2.5 with $\mathrm{p}<0.01$.

Intracranial pressure seems to be a crucial factor in developing CSF rhinorrhoea although in primary spontaneous cases it is not persistently high. Normal intracranial pressure is pulsatile and these pulsations imply continued brain perfusion. Normally the amplitude of cardiac pulse in the CSF pressure is $15 \mathrm{~mm}$ of water and combined respiratory and cardiac variation is about $45 \mathrm{~mm}$ of water ${ }^{10}$.

In CSF pressure curves it is seen that increased right atrial pressure increases the cerebral venous pressure and thus, transiently, increased CSF pressure. These pulsatile variations of CSF pressure are higher in obese subjects than their normal counterparts. Sugerman ${ }^{14,15}$ postulated that central obesity raises intra-abdominal pressure, consequently increasing the pleural pressure and cardiac filling pressure which in turn impedes the venous return from the brain leading to raised intracranial pressure. We speculate that the pulsatile variations of CSF pressure on the lamina cribriosa result in erosion of bone and protrusion of brain and/or meninges forming meningocele and/or encephalocele. Therein pulsatile pressure finally causes rupture of arachnoid without having support of underlying bone leading to formation of fistula. In our study we showed that four patients (5/15) with spontaneous CSF rhinorrhoea had an associated meningocele and/or encephalocele. These were recognised as a bluish polyp during nasendoscopy and confirmed histologically from the biopsy performed during the closure of the leak. The age of the study group of patients were from 37-70 years and the median was 50, these figures are very much similar to other studies $^{5,6}$

Why is it that females in their fifth and sixth decades are at an increased risk of developing CSF rhinorrhoea? The possible explanation may be that the female bones are thinner than the male making them prone to erosion and with advancing age the thickness of female cranial bones decreases $\left(\right.$ Lippert $\left.^{16}\right)$ whereas the thickness of male cranial bones increases with age, although this is not specific to the lamina cribrosa.

The percentage of spontaneous to total CSF rhinorrhoea is reported to be $3-4 \%$, but in our study we had a total 34 patients with CSF leak amongst them 15 were spontaneous cases and the percentage is therefore $44 \%$.

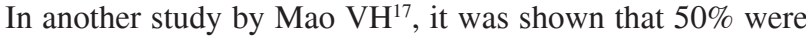
spontaneous (10 cases out of 20).

Dodson $\mathrm{EE}^{13}$, showed that $31 \%$ were spontaneous (9 spontaneous cases out of 29 cases). Hughes $\mathrm{GM}^{18}$, showed $35 \%$ were spontaneous (6 sp. Leaks out of 17 patients). It may a reflection of the small size of the study groups or possibly improvements in diagnostic techniques in nasendoscopy.

Much the largest group of CSF rhinorrhoea is traumatic $(80 \%)$ which heals with conservative treatment and does not warrant surgery.

Detection of beta-2 transferrin in the nasal fluid could be helpful to diagnose the presence of CSF leaks. In our series $80 \%$ patients were confirmed to have this protein in their nasal discharge. Although beta- 2 transferrin can be false positive in patients with inborn errors of metabolism of glycoprotein, chronic liver disease, genetic variants of $\operatorname{transferrin}^{19}$; some authors believe that venous blood sample should be collected simultaneously with the rhinorrhoea fluid, this was our practice.

Glucose estimation and oxidase test of the nasal fluid may also be helpful. Nasendoscopy in the outpatient clinics was done routinely to find the defect in the skull base but this could only be identified in $50 \%$ of cases. CT scans and/or MRI also detected the bony defect in $66 \%$ of patients, but it must be performed before any surgical intervention (especially coronal sections). We used intrathecal dyes isotope cysternogram only when there were difficulties in localising the defect through endoscopy or CT scan or in the case of recurrent leaks.

As a graft material we mainly used a combination of the turbinates (middle or inferior turbinate) and septal cartilage. Turbinates not only act as a unique composite graft; on removal of turbinate from the side of leak one has good exposure to the operative field. In the case of larger defects ( $>15 \mathrm{~mm}$ ), we reinforced the turbinates with fascia lata and muscles from the thigh. As tissue adhesive we mainly used tisseal glue and lyostypt with routine use of per-operative antibiotic and Bismuth \& Iodoform Paraffin Paste pack in the nose. 
In our series the success rate after initial repair was $80 \%$ and raised to $86 \%$ after second attempt. None of our patients suffered from post operative anosmia, transient hemi-paresis or frontal lobe abscess.

In both the diagnostic and operative steps the advent of the nasendoscope has played a key role in the management of CSF leaks. Previously the requirement for an intradural exposure frequently converted a small leak into an extensive one.

In conclusion our study has shown that obese middle aged women are more prone to spontaneous cerebrospinal fluid rhinorrhoea and that the surgical repair of choice is extracranial.

\section{References}

1. Beckhardt RN, Setzen M, Carras R. "Primary Spontaneous Cerebrospinal fluid Rhinorrhoea". Otolaryngol Head Neck Surg 1991;104:425-432.

2. Spetzler RF, Zambramski JM. "Cerebrospinal fluid fistulae: their management and repair". Youmans Neurosurgical Surgery, 3rd edition, 1990;4:2269-89.

3. Brisman R, Hughes JE, Mount LA. "Cerebrospinal fluid rhinorrhea and the empty sella". J Neurosurg. 1969;31:538-43.

4. Dohlman G. "Spontaneous cerebrospinal rhinorrhea". Acta Otolaryngol Suppl 1948;67:20-23.

5. Wigand ME. "Transnasal Ethmoidectomy Under Endoscopic Control”. Rhinology 1981;19:7-15.

6. Stankiewicz JA. "Complications in Endoscopic Intranasal Ethmoidectomy: an update". Laryngoscope 1989;99:686-90

7. Schechter MM, Rovit RL, Nelson K (1969). "Spontaneous high pressure CSF rhinorrhoea". Br J Radiol Aug 1969;42:619-22

8. Ommaya AK, Di Chiro G, Baldwin M, Pennybacker JB. "Non-traumatic cerebrospinal fluid rhinorrhoea". J Neurol Neurosurg Psychiatry 1968;3:214-25.

9. Schievink W, "Spontaneous CSF leaks: a review". Neurosurg Focus 2000;9:Article 8
10. Bradley, K. C. "Cerebrospinal Fluid Pressure". J. Neurol. Neurosurg. Psychiatry, 1970;33:387-397.

11. Badia L, Lougram S, Lund V. "Primary Spontaneous CSF Rhinorrhoea and Obesity". Am J Rhinol 2001;15:117-9.

12. Burns JA, Dodson EE, Gross CW. “Transnasal Endoscopic Repair of Craniofacial Fistula: A Refined Technique with Long Term Follow-up". Laryngoscope 1996;106:1080-1083.

13. Dodson EE, Gross CW, Swerddloff JL, Gustafson LN. "Transnasal Endoscopic Repair of CSF Rhinorrhoea and Skull base Defects: A Review of 29 Cases". Otolaryngology and Head-Neck Surgery; 1994;111: 600-5.

14. Sugerman HJ, Felton WL 3rd, Salvant JB Jr, Sismanis A, Kellum JM. "Effects of Surgically Induced Weight Loss on Idiopathic Intracranial Hypertension in Morbid Obesity". Neurology 1995; 45: 1655-1659.

15. Sugerman HJ, DeMaria EJ, Felton WL 3rd, Nakatsuka M, Sismanis A. "Increased Intracranial pressure and Cardiac Filling Pressure in Obesity-associated Pseudo tumour Cerebri”.

16. Lippert H, "Anatomie: Text u. Atlas: dt. u. latein. Namen”; 1975;72-76; ISBN:3541072113.

17. Mao VH, Keane WM, Atkins JP, Spiegel JR, Willcox TO, Rosen MR, Andrews D, Zwilleenberg D (1998). "Endoscopic Repair of CSF Rhinorrhoea". Otolaryngol Head Neck Surgery 2000;122:56-60.

18. Hughes RG, Jones NS, Robertson IJ (1996). “The Endoscopic Treatment of CSF Rhinorrhoea: The Nottingham Experience". The Journal of Larungology and Otology 1997;111:125-128.

19. Roelandse FW, van der Zwart N, Didden JH, van Loon J, Souverijn JH. Detection of CSF leakage by Iso electric Focusing on Polyacrylamide Gel, Direct Immunofixation of Transferrin and Silver Staining. Clinical Chemistry 1998;44:351-353. 